\title{
THREE DIMENSIONAL GREEN'S FUNCTION FOR SHIP MOTION AT FORWARD SPEED
}

\author{
MATIUR RAHMAN \\ Department of Applied Mathematics \\ Technical University of Nova Scotia \\ P.0. BOX 1000 \\ Halifax, Nova Scotia \\ Canada B3J $2 \times 4$
}

(Received December 12, 1988)

\begin{abstract}
The Green's function formulation for ship motion at forward speed contains double integrals with singularities in the path of integrations with respect to the wave number. In this study, the double integrals have been replaced by single integrals with the use of complex exponential integrals. It has been found that this analysis provides an efficient way of computing the wave resistance for three dimensional potential problem of ship motion with forward speed.
\end{abstract}

KEY WORDS AND PHRASES. Ship motion, Green's function, Hydrodynamics, Wave Resistance and Wave Responses.

\section{INTRODUCTION.}

In ship hydrodynamics, Green's functions play a very important role in predicting the wave resistance, wave induced responses at zero forward speed, and the motions of a vessel advancing in waves. The Green's function formulation for ship motions at forward speed is the most difficult part of the problem partly because it contains double integrals and partly because of the presence of the singularities in the path of integrations with respect to the wave numbers. Nowadays, considerable interest has been paid to evaluate the three dimensional Green's function for ship motions at forward speed.

Many authors including Haskind [1] and Havelock [2] have expressed the Green's function having a constant forward speed as a double integral. This form of Green's function is not suitable for numerical analysis because the detailed computation of the double integral is very expensive. Therefore, in the present study, we have replaced the double integral by a single integral (see Wu and Taylor [3]) involving a complex exponential integral, and it is found that it is more efficient to calculate the Green's function numerically.

2. A FORM OF THE GREEN'S FUNCTION.

Consider the coordinate system oxyz which is moving at constant forward speed U along the $x$ axis and $z$ measured positive upwards from the mean free surface (see Figure 1). 


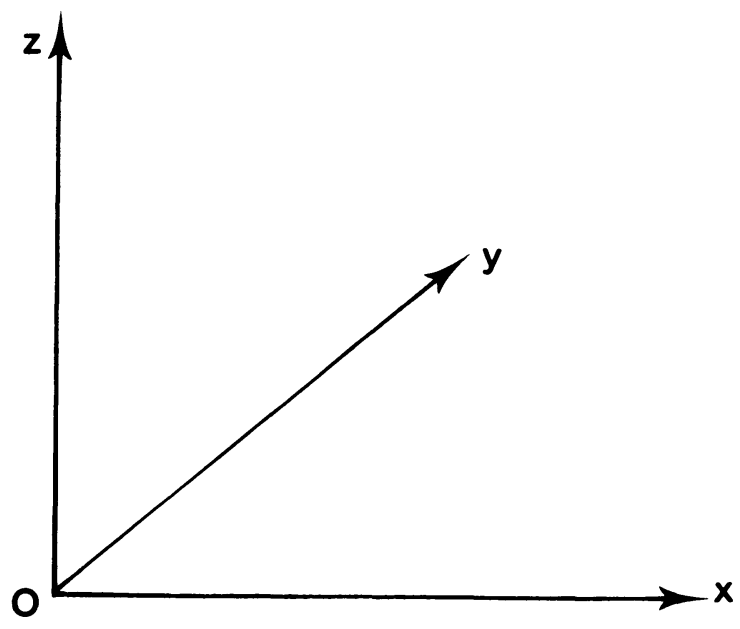

Figure 1: The Coodinate System

It is assumed that a ship is travelling at a constant forward speed $U$ along the $0 x$ direction and oscillating at a frequency $\omega$ as in the form of $e^{i \text { th }}$. Wehausen and Laitone [4] in 1960 have shown that the Green's function which satisfies the exact free surface condition can be written as

$$
\begin{aligned}
& G(x, y, z ; a, b, c)=\frac{1}{R}-\frac{1}{R_{1}}+\frac{2 g}{\pi} \int_{0}^{\gamma} d \theta \int_{0}^{\infty} F(\theta, k) d k+\frac{2 g}{\pi} \int_{\gamma}^{\pi / 2} d \theta \int_{L_{1}} F(\theta, k) d k \\
& +\frac{2 g}{\pi} \int_{\pi / 2}^{\pi} d \theta \int_{L_{2}} F(\theta, k) d k
\end{aligned}
$$

where

$$
\begin{aligned}
& R=\sqrt{(x-a)^{2}+(y-b)^{2}+(z-c)^{2}} \text { Rankine source located at }(a, b, c) \\
& R_{1}=\sqrt{(x-a)^{2}+(y-b)^{2}+(z+c)^{2}} \text { Image about the mean free surface at }(a, b,-c) \\
& g=\text { acceleration due to gravity } \\
& F(\theta, k)=\frac{k e^{k[(z+c)+i(x-a) \cos \theta]} \cos [k(y-b) \sin \theta]}{g k-(\omega+k U \cos \theta)^{2}}
\end{aligned}
$$

$(x, y, z)$ is the field point and $(a, b, c)$ is the source distribution point. The other parameters in Equation (2.1) are defined by

$$
\begin{array}{ll}
\gamma=0 & \text { if } \tau<\frac{1}{4} \\
\cos ^{-1}\left(\frac{1}{t \tau}\right) & \text { if } t>\frac{1}{4}
\end{array}
$$

where $\tau=\frac{\omega U}{g}$ is called the Strouhal number

The contours $\mathrm{L}_{1}$ and $\mathrm{L}_{2}$ are defined as follows: 


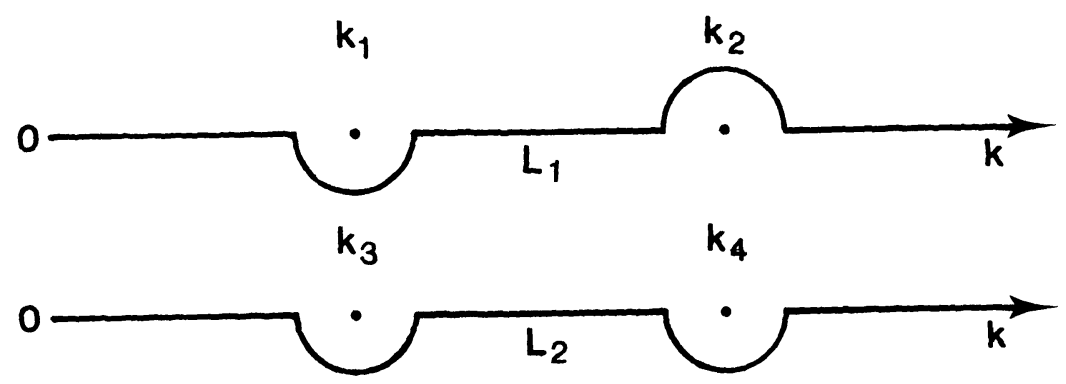

There are two singular points in $L_{1}$ and two singular points in the $L_{2}$ integral of Equation (2.1). These singular points can be obtained as follows:

$$
\begin{aligned}
& {\sqrt{g k_{1}}}_{1}, \overline{\sqrt{g k_{3}}}=\frac{1-\sqrt{1-4 \tau \cos \theta}}{2 \tau \cos \theta} \omega \\
& {\sqrt{g k_{2}}}_{2}, \overline{\sqrt{g k_{4}}}=\frac{1+\sqrt{1-4 \tau \cos \theta}}{2 \tau \cos \theta} \omega
\end{aligned}
$$

The alternative forms of these singularities $k_{1}, k_{2}, k_{3}$ and $k_{4}$ can be written as

$$
k_{2}, k_{1}=\frac{(1-2 \tau \cos \theta) \pm \sqrt{1-4 \tau \cos \theta}}{2 \tau^{2} \cos ^{2} \theta} v
$$

for $\pi / 2<\theta<\pi$; and where $v=\frac{\omega^{2}}{g}$.

It should be noted here that $k_{1}=k_{3}$ and $k_{2}=k_{4}$. These singularities are real in the ranges indicated. It is, however, worth mentioning here that in the range

$0<\theta<\gamma$, the singularities $k_{1}$ and $k_{2}$ become complex quantities and are either given by

or

$$
\sqrt{\mathrm{gk}_{2}}, \overline{\sqrt{\mathrm{gk}} 1}=\frac{1}{2 \tau \operatorname{i\sqrt {4\tau \operatorname {cos}\theta }-1}} \omega
$$

$$
k_{2}, k_{1}=\frac{1-2 \tau \cos \theta \pm i \sqrt{4 \tau \cos \theta-1}}{2 \tau^{2} \cos ^{2} \theta} v
$$

Thus the integrand in the integral

$$
\int_{0}^{\gamma}\left[\int_{0}^{\infty} F(\theta, k) d k\right] d \theta
$$

contains no real singular points in the path of integration from 0 to $\infty$.

\section{EVALUATION OF INTEGRALS.}

The Green's function given in equation (2.1) is difficult to integrate numerically because as we have seen in the previous section, the contours $L_{1}$ and $L_{2}$ have singularities at $k_{1}, k_{2}, k_{3}$ and $k_{4}$. This difficulty can be overcome by introducing the Cauchy Principal Value (PV) integrals.

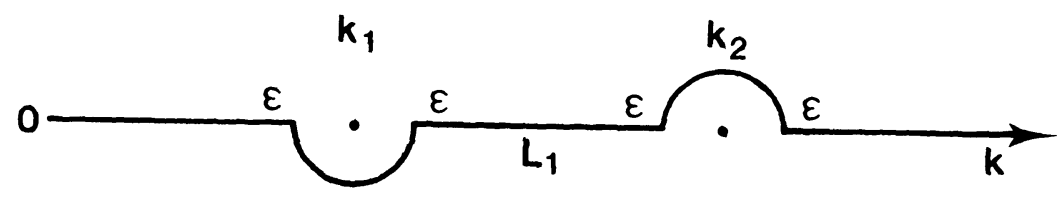


The first contour integral along the path $\mathrm{L}_{1}$ can be rewritten as

$$
\begin{aligned}
& G_{L_{1}}=\frac{2 g}{\pi} \int_{\gamma}^{\pi / 2} d \theta \int_{L_{1}} F(\theta, k) d k \\
& =\frac{2 g}{\pi} \int_{\gamma}^{\pi / 2} d \theta\left[\int_{0}^{k_{1}-\varepsilon}+\int_{-}+\int_{k_{1}+\varepsilon}^{k_{2}-\varepsilon}+\int_{k_{2}+\varepsilon}^{\infty} F(\theta, k) d k\right.
\end{aligned}
$$

When $\varepsilon+0$, equation (3.1) can be written as

where

$$
G_{L_{1}}=\frac{2 g}{\pi} \int_{\gamma}^{\pi / 2} d \theta\left(P . V_{.}\right) \quad \int_{0}^{\infty} F(\theta, k) d k+\frac{2 g}{\pi} \int_{\gamma}^{\pi / 2} d \theta\left\{\int_{T}+\int\right\} F(\theta, k) d k
$$

$$
\text { (P.v.) } \int_{0}^{\infty} F(\theta, k) d k=\operatorname{Lim}\left\{\int_{0}^{k_{1}-\varepsilon k_{2}-\varepsilon}+\int_{k_{1}+\varepsilon}+\int_{k_{2}+\varepsilon}^{\infty}\right\}(\theta, k) d k \varepsilon \rightarrow 0
$$

To evaluate the integral along the deformations $\checkmark$ and $\curvearrowright$, we decompose the integral $F(\theta, k)$ in terms of its singularities. We write

$$
F(\theta, k)=\frac{1}{g \sqrt{1-4 \tau \cos \theta}}\left[\frac{k_{1}}{k-k}-\frac{k_{2}}{k-k}\right] \text { exp }\{k[(z+c)+i(x-a) \cos \theta]\} \cos (k(y-b) \sin \theta)
$$

which can be put in the following compact form

$$
F(\theta, k)=\frac{1}{2 g \sqrt{1-4 \tau \cos \theta}}\left[\frac{k}{k-k_{1}}-\frac{k_{2}}{k-k_{2}}\right]\left[\exp \left(k x_{1}\right)+\exp \left(k x_{2}\right)\right]
$$

where

$$
\begin{aligned}
& x_{1}=(z+c)+i w_{+}, x_{2}=(z+c)+i w . \\
& w_{+}=(x-a) \cos \theta+(y-b) \sin \theta, \quad w_{-}=(x-a) \cos \theta-(y-b) \sin \theta
\end{aligned}
$$

We know that $(z+c)<0$ so we can redefine $x_{1}$ and $x_{2}$ as follows

$$
x_{1}=-\left\{(z+c)-i w_{+}\right\}, \quad x_{2}=-\left\{(z+c)-i w_{-}\right\}
$$

Thus, equation (3.5) can be rewritten as

$$
F(\theta, k)=\frac{1}{2 g \sqrt{1-4 \tau \cos \theta}}\left[\frac{k_{1}\left\{\exp \left(-k_{1}\right)+\exp \left(-k_{2}\right)\right\}}{k-k_{1}}-\frac{k_{2}\left\{\exp \left(-k_{1}\right)+\exp \left(-k_{2}\right)\right\}}{k-k_{2}}\right]
$$

The integration along the deformations $\checkmark$ and $\curvearrowright$ in equation (3.2) can be obtained according to the residue theorem. Thus

$$
\begin{gathered}
\left\{\int_{d}+\int_{\nu}\right\} F(\theta, k) d k \\
=\frac{\pi i}{2 g \sqrt{1-4 \tau \cos \theta}}\left\{k_{1}\left(\exp \left(-k_{1} x_{1}\right)+\exp \left(-k_{1} x_{2}\right)\right)+k_{2}\left(\exp \left(-k_{2} x_{1}\right)+\exp \left(-k_{2} x_{2}\right)\right)\right\}
\end{gathered}
$$

Thus, equation (3.9) reduces to 


$$
\begin{aligned}
& \mathrm{G}_{\mathrm{L}_{1}}=\frac{2 \mathrm{~g}}{\pi} \int_{\gamma}^{\pi / 2} \mathrm{~d} \theta(\mathrm{P} . \mathrm{V} .) \quad \int_{0}^{\infty} \mathrm{F}(\theta, \mathrm{k}) \mathrm{dk}
\end{aligned}
$$

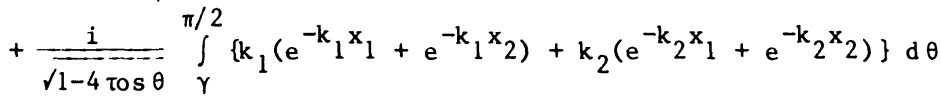

In a similar manner the second contour integral along path $\mathrm{L}_{2}$ equation (2.1) can be obtained

$$
\begin{aligned}
& \mathrm{G}_{\mathrm{L}_{2}}=\frac{2 \mathrm{~g}}{\pi} \int_{\pi / 2}^{\pi} \mathrm{d} \theta\left(\mathrm{P} \cdot \mathrm{V}_{.}\right) \int_{0}^{\infty} \mathrm{F}(\theta, \mathrm{k}) \mathrm{dk} \\
& +\frac{i}{\sqrt{1-4 \tau \cos \theta}} \int_{\pi / 2}^{\pi}\left\{k_{3}\left(e^{-k_{3} x_{1}}+e^{-k_{3} x_{2}}\right)-k_{4}\left(e^{-k_{4} x_{1}}+e^{-k_{4} x_{2}}\right)\right\}
\end{aligned}
$$

Therefore the Greens function in equation (2.1) can be rewritten as follows:

$$
\begin{aligned}
& G_{1}(x, y, z ; a, b, c)=\frac{1}{R}-\frac{1}{R_{1}}+\frac{2 g}{\pi} \int_{0}^{\gamma} d \theta \int_{0}^{\infty} F(\theta, k) d k \\
& +\frac{2 g}{\pi}\left\{\int_{\gamma}^{\pi / 2}+\int_{\pi / 2}^{\pi}\right\} d \theta(P . V .) \int_{0}^{\infty} F(\theta, k) d k \\
& +\frac{i}{\sqrt{1-4 \tau \cos \theta}}\left\{\int _ { \gamma } ^ { \pi / 2 } \left\{k_{1}\left(e^{-k_{1} x_{1}}+e^{\left.-k_{1} x_{2}\right)}+k_{2}\left(e^{-k_{2} x_{1}}+e^{-k_{2} x_{2}}\right)\right\} d \theta\right.\right. \\
& +\int_{\pi / 2}^{\pi}\left\{k _ { 3 } \left(e^{-k_{3} x_{1}} d e^{\left.-k_{3} x_{2}\right)}-k_{4}\left(e^{\left.\left.-k_{4} x_{1}+e^{\left.-k_{4} x_{2}\right)}\right\} d \theta\right\}}\right.\right.\right.
\end{aligned}
$$

or,

$$
G=G_{1}+G_{2}+G_{3}+G_{4}+i G_{5}
$$

where

$$
\begin{aligned}
& G_{1}=\frac{1}{R}, G_{2}=-\frac{1}{R_{1}} \\
& G_{3}=\frac{2 g}{\pi} \int_{0}^{\gamma} d \theta \int_{0}^{\infty} F(\theta, k) d k \quad G_{4}=\frac{2 g}{\pi}\left\{\int_{\gamma}^{\pi / 2}+\int_{\pi / 2}^{\pi}\right\} d \theta(P . V .) \int_{0}^{\infty} F(\theta, k) d k \\
& G_{5}=\int_{\gamma}^{\pi / 2} \frac{1}{\sqrt{1-4 \tau \cos \theta}}\left\{k _ { 1 } \left(e^{-k_{1} x_{1}}+e^{\left.-k_{1} x_{2}\right)}+k_{2}\left(e^{-k_{2} x_{1}}+e^{\left.\left.-k_{2} x_{2}\right)\right\} d \theta}\right.\right.\right. \\
& +\int_{\pi / 2}^{\pi} \frac{1}{\sqrt{1-4 \tau \cos \theta}}\left\{k _ { 3 } \left(e^{-k_{3} x_{1}} d e^{\left.\left.-k_{3} x_{2}\right)-k_{4}\left(e^{-k_{4} x_{1}}+e^{-k_{4} x_{2}}\right)\right\} d \theta}\right.\right.
\end{aligned}
$$

There are two cases to be considered.

Case I

$$
\gamma=0 \quad \text { if } \tau<\frac{1}{4}
$$

and in this case $G_{3}=0$.

Therefore, equation (3.13) becomes

$$
G=G_{1}+G_{2}+G_{4}+i G_{5}
$$


Case II

$$
\gamma=\cos ^{-1} \frac{1}{4 \tau} \quad \text { if } \tau>\frac{1}{4}
$$

and in this case equation (3.13) becomes

$$
G=G_{1}+G_{2}+G_{3}+G_{4}+i G_{5}
$$

The double integrals in $G_{3}$ and $G_{4}$ are highly oscillatory at large values of $k$ because of the imaginary argument of the exponential function. In order to calculate them numerically, at minimum computer cost, these integrals must be reduced to single integrals as suggested by Shen and Farell [5], and Inglis and Price [6]. We shall treat Case I first and evaluate the Cauchy Principal Value (P.V.) integral in $G_{4^{\circ}}$

The term $G_{4}$ of the Green function can be written as

$$
G_{4}=\frac{1}{\pi} \int_{\gamma}^{\pi / 2} \frac{d \theta}{\sqrt{1-4 \tau \cos \theta}}\left(I_{1}+I_{2}-I_{3}-I_{4}\right) \quad+\frac{1}{\pi} \int_{\pi / 2}^{\pi} \frac{d \theta}{\sqrt{1-4 \tau \cos \theta}}\left(I_{5}+I_{6}-I_{7}-I_{8}\right)
$$

where

$$
\begin{aligned}
& I_{1}=\text { (P.V.) } \int_{0}^{\infty} \frac{k_{1} \exp \left(-k_{1}\right)}{k-k_{1}} d k, \quad I_{2}=\left(P . V_{.}\right) \int_{0}^{\infty} \frac{k_{1} \exp \left(-k x_{2}\right)}{k-k_{1}} d k \\
& I_{3}=\text { (P.V.) } \int_{0}^{\infty} \frac{k_{2} \exp \left(-k_{1}\right)}{k-k_{2}} d k, \quad I_{4}=\text { (P.V.) } \int_{0}^{\infty} \frac{k_{2} \exp \left(-k x_{2}\right)}{k-k_{2}} d k
\end{aligned}
$$

for $\gamma<\theta<\pi / 2$

$$
\begin{aligned}
& I_{5}=(P . V .) \quad \int_{0}^{\infty} \frac{k_{2} \exp \left(-k x_{1}\right)}{k-k_{3}} d k, \quad I_{6}=(P . V .) \int_{0}^{\infty} \frac{k_{3} \exp \left(-k x_{1}\right)}{k-k_{3}} d k \\
& I_{7}=(P . V .) \int_{0}^{\infty} \frac{k_{4} \exp \left(-k x_{1}\right)}{k-k_{4}} d k \quad I_{8}=\left(P . V_{.}\right) \int_{0}^{\infty} \frac{k_{4} \exp \left(-k x_{2}\right)}{k-k_{4}} d k
\end{aligned}
$$

for $\pi / 2<\theta<\pi$.

To obtain analytic expressions of these integrals, we consider a contour in the $K=k+i k$ ' plane as suggested by Smith et al [7] (see Figure 2) and later used by Chen et al [8].

We impose the condition that

$$
I_{m}\left[-K x_{1}\right]=0
$$

on the integration path 5 which makes an angle $\alpha$ with the real axis, so that the argument of the exponential can be made real along the ray.

Therefore, we get

$$
I_{m}\left[-\left(k+i k^{\prime}\right)\left(|z+c|-i w_{+}\right)\right]=0
$$

which simplifies to yield

and

$$
{\frac{k^{\prime}}{k}}^{\prime}=\frac{\mathbf{w}_{+}}{|\mathbf{z}+\mathrm{c}|}
$$

$$
\alpha=\tan ^{-1} \frac{k^{\prime}}{k}=\tan ^{-1} \frac{w_{+}}{|z+c|}
$$




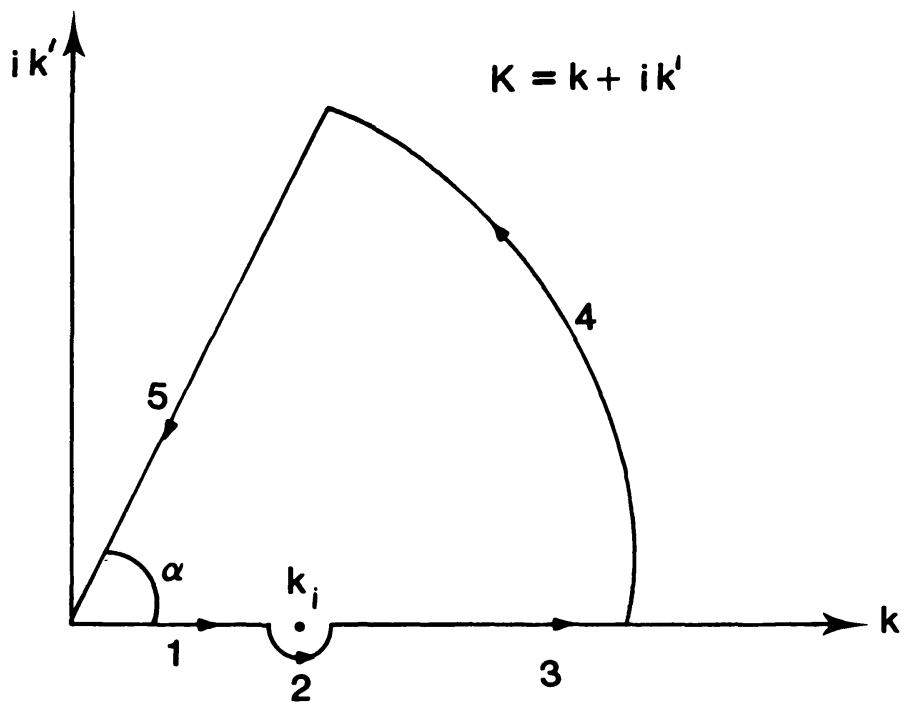

Figure 2: A closed contour for $w_{+}>0$

Thus with this value of $\alpha$,

$$
R_{e}\left[-\left(k+i k^{\prime}\right)\left(|z+c|-i w_{+}\right)\right]=-k \frac{|z+c|^{2}+w_{+}^{2}}{|z+c|}=-k v<0
$$

Also, we have

$$
k=k+i k^{\prime} \quad=\frac{k V}{|z+c|-i w_{+}}
$$

Integrating along the contour shown in Figure 2,

$$
\begin{aligned}
& I_{1}=2 \pi i\left\{k_{1} \exp \left(-k_{1} x_{1}\right)\right\}-\pi i k_{1} \exp \left(-k_{1} x_{1}\right)-\int_{5} \frac{k_{1} \exp (-k x)}{k-k_{1}} d k \\
& =(\pi i) k_{1} \exp \left(-k_{1} x_{1}\right)-\int_{5} \frac{k_{1} \exp \left(-k_{1}\right)}{k-k_{1}} d k
\end{aligned}
$$

Along the path 5

$$
\begin{aligned}
& \int_{5}=k_{1} \int_{\infty}^{0} \frac{\exp (-k V)}{(k V)-k_{1} x_{1}} d(k v)=-k_{1} \int_{0}^{\infty} \frac{\exp (-u)}{u-k_{1} x_{1}} d u \\
& =-k_{1} \exp \left(-k_{1} x_{1}\right) E_{1}\left(-k_{1} x_{1}\right)
\end{aligned}
$$

where

$$
E_{1}(-z)=\int_{-z}^{\infty} \frac{e^{-t}}{t} d t,|\arg (z)|<\pi=\text { exponential integral. }
$$

Therefore, for $w_{+}>0$,

$$
I_{1}=k_{1} \exp \left(-k_{1} x_{1}\right)\left\{E_{1}-\left(k_{1} x_{1}\right)+\pi i\right\}
$$

It is to be noted here that for $w_{+}<0$ the contour will be as follows: 


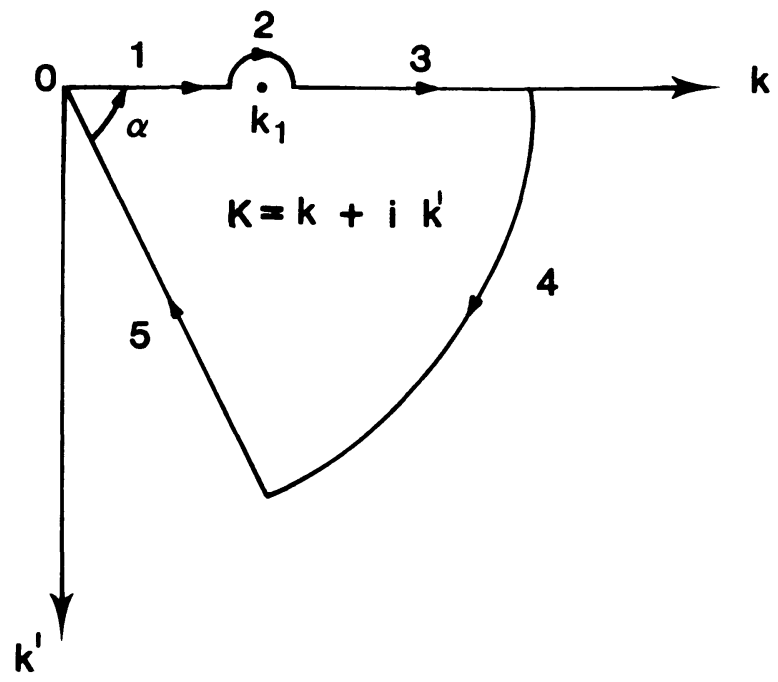

Figure 3: Closed contour for $w_{+}<0$

Thus

$$
I_{1}=k_{1} \exp \left(-k_{1} x_{1}\right)\left\{E_{1}\left(-k_{1} x_{1}\right)-\pi i\right\}
$$

Also, for $w_{+}=0$,

$$
I_{1}=k_{1} \exp \left(-k_{1} x_{1}\right)\left\{E_{1}\left(-k_{1} x_{1}\right)+\pi i\right\}
$$

which is obtained using the following definitions (see Abramowitz and Stegun [9] 1965, p. 228).

$$
E_{1}(-x+i 0)=\left(\text { P.V. ) } \int_{-x}^{\infty} \frac{e^{-t}}{t} d t-\pi i, E_{1}(-x-10)=\text { (P.V.) } \int_{-x}^{\infty} \frac{e^{-t}}{t} d t+\pi i\right.
$$

such that for $w_{+}=0$

$$
I_{1}=k_{1} \exp \left(-k_{2} x_{1}\right) \quad\left[E_{1}\left(-k_{1} x_{1}\right)+\pi f\right]
$$

Similarly, we can calculate the other integrals. Thus summing up the situation, we get for $\quad w_{+}>0$ or $I_{m}\left(-k_{1} x_{1}\right)>0$

$$
I_{1}=k_{1} \exp \left(-k_{1} x_{1}\right)\left\{E_{1}\left(-k_{1} x_{1}\right)+\pi i\right\}
$$

and for $w_{+}<0$ or $I_{m}\left(-k_{1} x_{1}\right)<0$

$$
I_{1}=k_{1} \exp \left(-k_{1} x_{1}\right)\left\{E_{1}\left(-k_{1} x_{1}\right)-\pi i\right)
$$

Similarly,

$$
\begin{aligned}
I_{2}= & k_{1} \exp \left(-k_{1} x_{2}\right)\left[E_{1}\left(-k_{1} x_{2}\right)+\pi i\right], \text { for } I_{m}\left(-k_{1} x_{2}\right)>0 \\
& k_{1} \exp \left(-k_{1} x_{2}\right)\left[E_{1}\left(-k_{1} x_{2}\right)-\pi i\right], \text { for } I_{m}\left(-k_{1} x_{2}\right)<0 \\
I_{3}= & k_{2} \exp \left(-k_{2} x_{1}\right)\left[E_{1}\left(-k_{2} x_{1}\right)-\pi i\right], \text { for } I_{m}\left(-k_{2} x_{1}\right)>0 \\
& k_{2} \exp \left(-k_{2} x_{1}\right)\left[E_{1}\left(-k_{2} x_{1}\right)+\pi i\right], \text { for } I_{m}\left(-k_{2} x_{1}\right)<0
\end{aligned}
$$




$$
\begin{aligned}
I_{4}= & k_{2} \exp \left(-k_{2} x_{2}\right)\left[E_{1}\left(-k_{2} x_{2}\right)-\pi i\right], \text { for } I_{m}\left(-k_{2} x_{2}\right)>0 \\
& k_{2} \exp \left(-k_{2} x_{2}\right)\left[E_{1}\left(-k_{2} x_{2}\right)+\pi i\right], \text { for } I_{m}\left(-k_{2} x_{2}\right)<0 \\
I_{5}= & k_{3} \exp \left(-k_{3} x_{1}\right)\left[E_{1}\left(-k_{2} x_{1}\right)+\pi i\right], \text { for } I_{m}\left(-k_{3} x_{1}\right)>0 \\
& k_{3} \exp \left(-k_{3} x_{1}\right)\left[E_{1}\left(-k_{3} x_{1}\right)-\pi i\right], \text { for } I_{m}\left(-k_{3} x_{1}\right)<0 \\
I_{6}= & k_{3} \exp \left(-k_{3} x_{2}\right)\left[E_{1}\left(-k_{3} x_{2}\right)+\pi i\right], \text { for } I_{m}\left(-k_{3} x_{2}\right)>0 \\
& k_{3} \exp \left(-k_{3} x_{2}\right)\left[E_{1}\left(-k_{3} x_{2}\right)-\pi i\right], \text { for } I_{m}\left(-k_{3} x_{2}\right)<0 \\
I_{7}= & k_{4} \exp \left(-k_{4} x_{1}\right)\left[E_{1}\left(-k_{4} x_{1}\right)+\pi i\right], \text { for } I_{m}\left(-k_{4} x_{1}\right)>0 \\
& k_{4} \exp \left(-k_{4} x_{1}\right)\left[E_{1}\left(-k_{4} x_{1}\right)-\pi i\right], \text { for } I_{m}\left(-k_{4} x_{1}\right)<0 \\
I_{8}= & k_{4} \exp \left(-k_{4} x_{2}\right)\left[E_{1}\left(-k_{4} x_{2}\right)+\pi i\right], \text { for } I_{m}\left(-k_{4} x_{2}\right)>0 \\
& k_{4} \exp \left(-k_{4} x_{4}\right)\left[E_{1}\left(-k_{4} x_{2}\right)-\pi i\right], \text { for } I_{m}\left(-k_{4} x_{2}\right)<0
\end{aligned}
$$

Now adding the terms in $G_{4}$ and $G_{5}$ given by the equations (3.17) and (3.14), respectively, we obtain

$$
\begin{aligned}
& G_{4}+i G_{5}=\frac{1}{\pi} \int_{\gamma}^{\pi / 2} \frac{1}{\sqrt{1-4 \tau \cos \theta}}\left\{I_{1}+I_{2}-I_{3}-I_{4}+\pi i\left(I_{11}+I_{12}+I_{21}+I_{22}\right)\right\} d \theta \\
& +\frac{1}{\pi} \int_{\pi / 2}^{\pi} \frac{1}{\sqrt{1-4 \pi \cos \theta}}\left\{\left(I_{5}+I_{6}-I_{7}-I_{8}\right)+\pi i\left(I_{31}+I_{32}-I_{41}-I_{42}\right)\right\} d \theta
\end{aligned}
$$

where

$$
I_{i j}=k_{i} \exp \left(-k_{i} x_{j}\right), j=1,2,3,4 ; j=1,2 \text {. }
$$

Thus, if we combine the corresponding integrands of $G_{4}+i G_{5}$, we obtain First Integral $=\frac{1}{\pi} \int_{\gamma}^{\pi / 2} \frac{k_{1} \exp \left(-k_{1} x_{1}\right)}{\sqrt{1-4 \tau \cos \theta}}\left[E_{1}\left(-k_{1} x_{1}\right)+2 \pi i\right] d \theta, \quad$ for $I_{m}\left(-k_{1} x_{1}\right)>0$

$$
\frac{1}{\pi} \int_{\gamma}^{\pi / 2} \frac{k_{1} \exp \left(-k_{1} x_{1}\right)}{\sqrt{1-4 \tau \cos \theta}} E_{1}\left(-k_{1} x_{1}\right) d \theta, \quad \text { for } I_{m}\left(-k_{1} x_{1}\right)<0
$$

Second Integral $=\frac{1}{\pi} \int_{\gamma}^{\pi / 2} \frac{k_{1} \exp \left(-k_{1} x_{2}\right)}{\sqrt{1-4 \tau \cos \theta}}\left[E_{1}\left(-k_{1} x_{2}\right)+2 \pi i\right] d \theta, \quad$ for $I_{m}\left(-k_{1} x_{2}\right)>0$

$$
\frac{1}{\pi} \int_{\gamma}^{\pi / 2} \frac{k_{1} \exp \left(-k_{1} x_{2}\right)}{\sqrt{1-4 \tau \cos \theta}} E_{1}\left(-k_{1} x_{2}\right) d \theta, \quad \text { for } I_{m}\left(-k_{1} x_{2}\right)<0
$$




$$
\begin{aligned}
\text { Third Integral }= & \frac{1}{\pi} \int_{\gamma}^{\pi / 2} \frac{k_{2} \exp \left(-k_{2} x_{1}\right)}{\sqrt{1-4} \tau \cos \theta}\left[-E_{1}\left(-k_{2} x_{1}\right)+2 \pi i\right] d \theta, & & \text { for } I_{m}\left(-k_{2} x_{1}\right)>0 \\
& -\frac{1}{\pi} \int_{\gamma}^{\pi / 2} \frac{k_{2} \exp \left(-k_{2} x_{1}\right)}{\sqrt{1-4} \operatorname{tcos} \theta} E_{1}\left(-k_{2} x_{1}\right) d \theta, & & \text { for } I_{m}\left(-k_{2} x_{1}\right)<0
\end{aligned}
$$

Fourth Integral $=\frac{1}{\pi} \int_{\gamma}^{\pi / 2} \frac{k_{2} \exp \left(-k_{2} x_{2}\right)}{\sqrt{1-4 \tau \cos \theta}}\left[-E_{1}\left(-k_{2} x_{2}\right)+2 \pi\right] d \theta, \quad$ for $I_{m}\left(-k_{2} x_{2}\right)<0$

$$
-\frac{1}{\pi} \int_{\gamma}^{\pi / 2} \frac{k_{2} \exp \left(-k_{2} x_{2}\right)}{\sqrt{1-4 \tau \cos \theta}} E_{1}\left(-k_{2} x_{2}\right) d \theta, \quad \text { for } I_{m}\left(-k_{2} x_{2}\right)<0
$$

Fifth Integra1 $=\frac{1}{\pi} \int_{\pi / 2}^{\pi} \frac{k_{3} \exp \left(-k_{3} x_{1}\right)}{\sqrt{1-4 \tau \cos \theta}}\left[E_{1}\left(-k_{3} x_{1}\right)+2 \pi i\right] d \theta, \quad$ for $I_{m}\left(-k_{3} x_{1}\right)>0$

$$
\frac{1}{\pi} \int_{\pi / 2}^{\pi} \frac{k_{3} \exp \left(-k_{3} x_{2}\right)}{\sqrt{1-4 \tau \cos \theta}} E_{1}\left(-k_{3} x_{1}\right) d \theta, \quad \text { for } I_{m}\left(-k_{3} x_{1}\right)<0
$$

Sixth Integral $=\frac{1}{\pi} \int_{\pi / 2}^{\pi} \frac{k_{3} \exp \left(-k_{3} x_{2}\right)}{\sqrt{1-4 \tau \cos \theta}}\left[E_{1}\left(-k_{3} x_{2}\right)+2 \pi i\right] d \theta, \quad$ for $I_{m}\left(-k_{3} x_{2}\right)<0$

$$
\frac{1}{\pi} \int_{\pi / 2}^{\pi} \frac{k_{3} \exp \left(-k_{3} x_{2}\right)}{\sqrt{1-4 \tau \cos \theta}} E_{1}\left(-k_{3} x_{2}\right) d \theta, \quad \text { for } I_{m}\left(-k_{3} x_{2}\right)<0
$$

Seventh Integral $=\frac{1}{\pi} \int_{\pi / 2}^{\pi / 2} \frac{k_{4} \exp \left(-k_{4} x_{1}\right)}{\sqrt{1-4 \tau \cos \theta}}\left[-E_{1}\left(-k_{4} x_{1}\right)-2 \pi i\right] d \theta, \quad$ for $I_{m}\left(-k_{4} x_{1}\right)>0$

$$
\frac{1}{\pi} \int_{\pi / 2}^{\pi} \frac{k_{4} \exp \left(-k_{4} x_{4}\right)}{\sqrt{1-4} \tau \cos \theta}\left[E_{1}\left(-k_{4} x_{1}\right) d \theta, \quad \text { for } I_{m}\left(-k_{4} x_{1}\right)<0\right.
$$

Eighth Integral $=\frac{1}{\pi} \int_{\pi / 2}^{\pi} \frac{k_{4} \exp \left(-k_{4} x_{2}\right)}{\sqrt{1-4 \tau \cos \theta}}\left[-E_{1}\left(-k_{4} x_{2}\right)-2 \pi i\right] d \theta, \quad$ for $I_{m}\left(-k_{4} x_{2}\right)>0$

$$
-\frac{1}{\pi} \int_{\pi / 2}^{\pi} \frac{k_{4} \exp \left(-k_{4} x_{2}\right)}{\sqrt{1-4 \tau \cos \theta}} E_{1}\left(-k_{4} x_{2}\right) d \theta, \quad \text { for } I_{m}\left(-k_{4} x_{2}\right)<0
$$

Therefore, for Case I, we can evaluate the Green's function given in equation (3.15).

To evaluate the Green's function for Case II given in equation (3.16), we need to express the $G_{3}$ term in exponential integrals as given below:

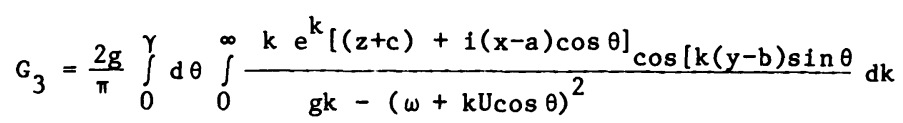




$$
=\frac{1}{\pi i} \int_{0}^{\gamma} \frac{1}{\sqrt{4} \tau \cos \theta-1}\left(\mathrm{~J}_{1}+\mathrm{J}_{2}-\mathrm{J}_{3}-\mathrm{J}_{4}\right) \mathrm{d} \theta
$$

where

$$
\begin{aligned}
& \mathrm{J}_{1}=\int_{0}^{\infty} \frac{\mathrm{k}_{1}}{\mathrm{k}-\mathrm{k}_{1}} \exp \left(-\mathrm{kx}_{1}\right) \mathrm{dk}, \mathrm{J}_{2}=\int_{0}^{\infty} \frac{\mathrm{k}_{1}}{\mathrm{k}-\mathrm{k}_{1}} \exp \left(-\mathrm{kx}_{2}\right) \mathrm{dk} \\
& \mathrm{J}_{3}=\int_{0}^{\infty} \frac{\mathrm{k}_{2}}{\mathrm{k}-\mathrm{k}_{2}} \exp \left(-\mathrm{kx}_{1}\right) \mathrm{dk}, \mathrm{J}_{4}=\int_{0}^{\infty} \frac{\mathrm{k}_{2}}{\mathrm{k}-\mathrm{k}_{2}} \exp \left(-\mathrm{kx}_{2}\right) \mathrm{dk}
\end{aligned}
$$

and $k_{1}$ and $k_{2}$ are the complex roots of

$$
g k-(\omega+k U \cos \theta)^{2}=0
$$

Using the contour in Figure 2, it can be easily shown that

$$
\begin{aligned}
& J_{1}=k_{1} \exp \left(-k_{1} x_{1}\right) E_{1}\left(-k_{1} x_{1}\right) \quad I_{m}\left(-k_{1} x_{1}\right)>0 \\
& k_{1} \exp \left(-k_{1} x_{1}\right)\left[E_{1}\left(-k_{1} x_{1}\right)-2 \pi i\right] I_{m}\left(-k_{1} x_{1}\right)<0
\end{aligned}
$$

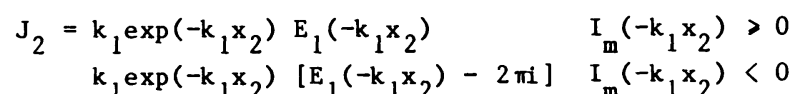

$$
\begin{aligned}
& \mathrm{J}_{3}=\mathrm{k}_{2} \exp \left(-\mathrm{k}_{2} \mathrm{x}_{1}\right) \mathrm{E}_{1}\left(-\mathrm{k}_{2} \mathrm{x}_{1}\right) \quad \mathrm{I}_{\mathrm{m}}\left(-\mathrm{k}_{2} \mathrm{x}_{1}\right)>0 \\
& k_{2} \exp \left(-k_{2} x_{1}\right)\left[E_{1}\left(-k_{2} x_{1}\right)-2 \pi i\right] \quad I_{m}\left(-k_{2} x_{1}\right)<0 \\
& J_{4}=k_{2} \exp \left(-k_{2} x_{2}\right)\left[E_{1}\left(-k_{2} x_{2}\right)+2 \pi i\right] \quad I_{m}\left(-k_{2} x_{2}\right)>0 \\
& -k \times) E(-k x)
\end{aligned}
$$

Thus, with this information, we can evaluate the Green's function for Case II from equation (3.16).

4. RESULTS AND CONCLUSiONS.

The present form of Green's function is equivalent to that used by Wu and Taylor, but in a different form. The terms $G_{1}, G_{2}$ and $G_{3}$ are all identical to those used by Chen et al [8]. However, in the present study, we have combined the $G_{4}$ and $G_{5}$ terms to correspond with the form of Wu and Taylor. It appears that our studies are quite similar to those of Wu and Taylor, and Chen et al.

The double integral arising in the evaluation of Green's function has been replaced by a single integral with the use of complex exponential integrals. The present work has provided an alternative form but similar to that of Wu and Taylor, and has been found to be efficient for the analysis of the three dimensional potential problem of ship motion with forward speed. 
ACKNOWLEDGEMENTS. This work has been performed under Contract No. OSC87-00549-(010) with the Defence Research Establishment Atlantic while the author was on Sabbatical Leave from the Technical University of Nova Scotia.

\section{REFERENCES}

1. HASKIND, M.D., The Hydrodynamic Theory of Ship Oscillations in Rolling and Pitching, Prik. Mat. Mekh. 10 (1946), 33-66.

2. HAVELOCK, T.H., The Effect of Speed of Advance Upon the Damping of Heave and Pitch, Trans. Inst. Nava1 Architect, 100, (1958), 131-135.

3. WU, G.X. and TAYLOR, R.E., A Green's Function Form for Ship Motions at Forward Speed, International Ship Progress 34 (1987), 189-196.

4. WEHAUSEN, J.V. and LAITONE, E.V. Surface Waves, Handbuch der Physik $\underline{9}$ (1960), 446-778.

5. SHEN, H.T. and FARELL, C., Numerical Calculation of the Wave Integrals in the Linearized Theory of Water Waves, Journal of Ship Research 21 (1977).

6. INGLIS, R.B. and PRICE, W.G., Calculation of the Velocity Potential of a Translating, Pulsating Source, Transaction, RINA, 1980.

7. SMITH, A.M.D., GIESING, J.P. and HESS, J.L., Calculation of Waves and Wave Resistance for Bodies Moving on or Beneath the Surface of the Sea, Douglas Aircraft Company Report 31488A, 1963.

8. CHEN, H.H., TORNG, J.M. and SHIN, Y.S., Formulation, Method of Solution and Procedures for Hydrodynamic Pressure Project, Technical Report RD-85026, November 1985.

9. ABRAMOWITZ, M. and STEGUN, A., Handbook of Mathematical Functions, Dover Publications, 1965. 


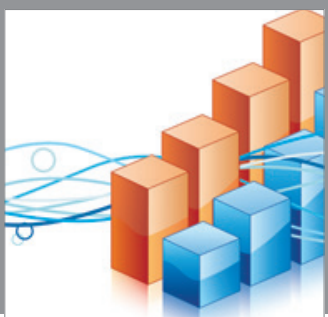

Advances in

Operations Research

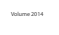

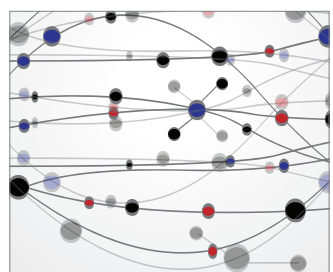

\section{The Scientific} World Journal
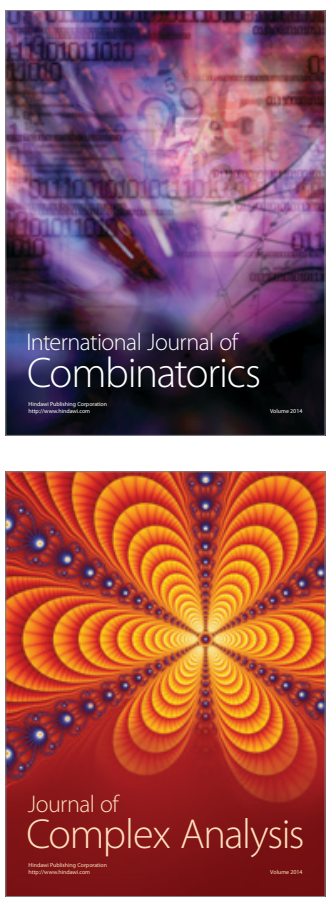

International Journal of

Mathematics and

Mathematical

Sciences
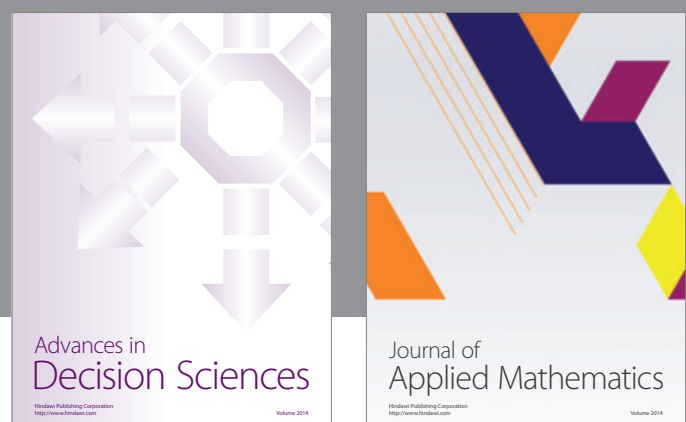

Journal of

Applied Mathematics
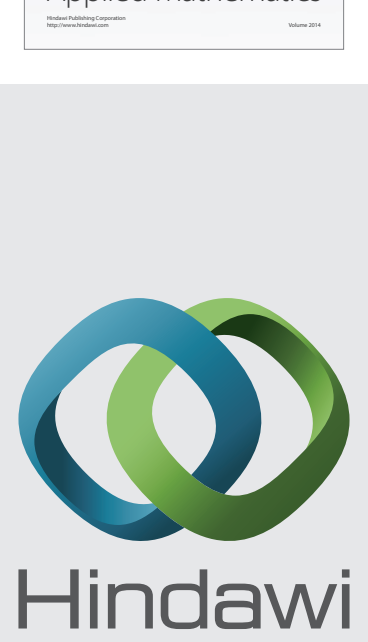

Submit your manuscripts at http://www.hindawi.com
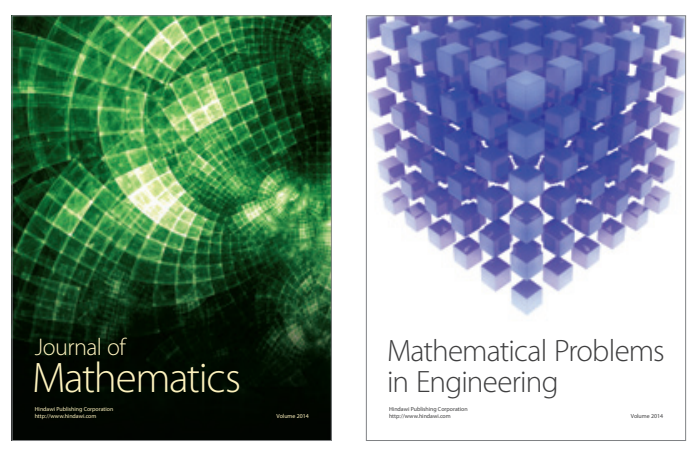

Mathematical Problems in Engineering
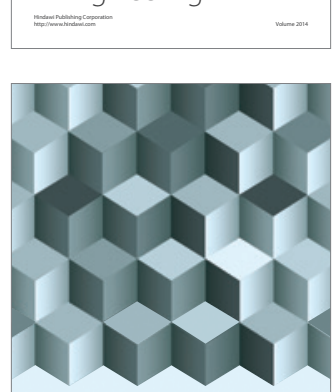

Journal of

Function Spaces
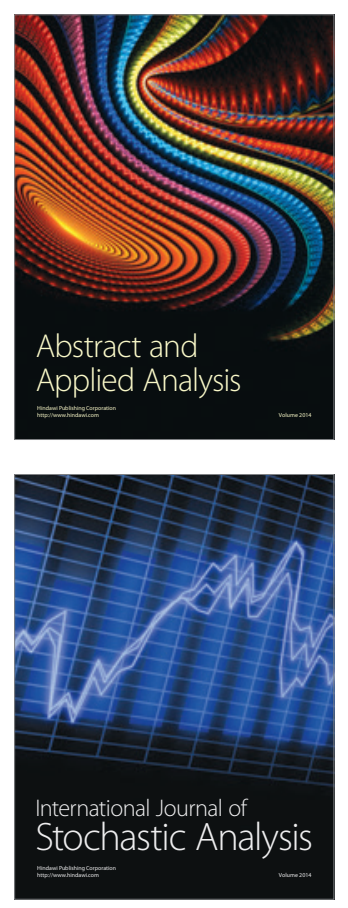

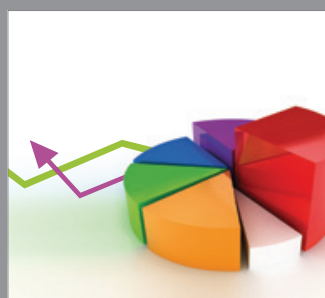

ournal of

Probability and Statistics

Promensencen
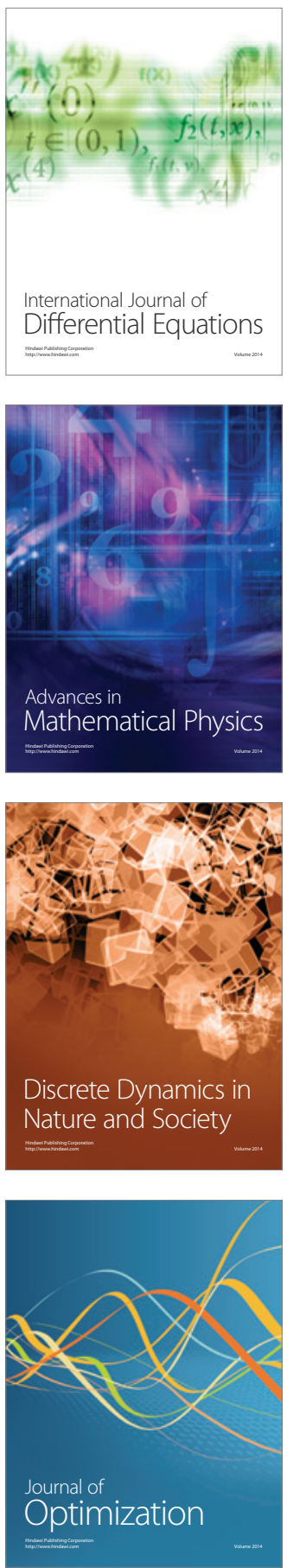